\title{
Vanadium-Substituted Wells-Dawson Potassium Salt as Catalyst for Liq- uid phase Oxidation of 1,4-dihydropyridine Derivative
}

\author{
Laura M. Sanchez ${ }^{1, *}$, Ángel G. Sathicq ${ }^{1}$, Graciela T. Baronetti ${ }^{2}$ and Horacio J. Thomas ${ }^{1,3}$

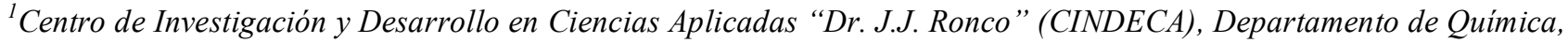 \\ Facultad de Ciencias Exactas, UNLP-CCT-CONICET. Calles 47 N 257, B1900AJK La Plata, Argentina \\ ${ }^{2}$ Departamento de Ingeniería Química, Facultad de Ingeniería, Universidad de Buenos Aires, Ciudad Universitaria, \\ C1428BG Buenos Aires, Argentina \\ ${ }^{3}$ Planta Piloto Multipropósito PlaPiMu, UNLP-CICPBA, Camino Centenario y 508, CP1987, Manuel B. Gonnet, Ar- \\ gentina
}

\begin{abstract}
Dawson-type heteropolyoxometalate compounds (HPC) $\mathrm{P}_{2} \mathrm{M}_{18} \mathrm{O}_{62}{ }^{-6}(\mathrm{M}=\mathrm{Mo}$, W) have received increasing attention in the catalytic field due to the combination of redox and acidic properties in the same structure. It has been proved that the introduction of vanadium into the Keggin framework is beneficial for redox catalysis, shifting its activity from acid to redox-dominated.

In this work we prepared and characterized vanadium (V) substituted Wells-Dawson heteropolysalt (WDKV). Fresh solid samples were characterized by ${ }^{31} \mathrm{P}$ MAS-NMR, FTIR, SEM, XRD, TGA and Potentiometric titration measurements. Also, we performed the oxidation of a 1,4-dihydropyridine using WDKV as catalyst in acetonitrile media, with $\mathrm{H}_{2} \mathrm{O}_{2}$ as oxidant agent. The optimal procedure is the following: $1 \mathrm{mmol} \%$ of WDKV, a ratio 1,4-DHP: $\mathrm{H}_{2} \mathrm{O}_{2}(1: 214)$ at reflux of acetonitrile. It must be noted that along all reactions, no secondary products were observed.
\end{abstract}

Keywords: Heteropolycompound, Oxidation, Hydrogen peroxide. 1,4-dihydropyridine.

\section{INTRODUCTION}

Dawson-type heteropolyoxometalate compounds (HPCs) $\mathrm{P}_{2} \mathrm{M}_{18} \mathrm{O}_{62}{ }^{-6}(\mathrm{M}=\mathrm{Mo}, \mathrm{W})$ have received increasing attention in the catalytic field due to the combination of redox and acidic properties in the same structure. As a result, HPCs can be useful homogeneous and heterogeneous catalysts for acidbase and oxidation reactions. One of the great advantages of HPCs catalysts is the possibility of making a priori design of the compound formulation, 'tailoring' the composition so as to introduce those elements that are necessary to achieve defined properties [1].

1,4-dihydropyridines (1,4-DHPs) are a class of heterocyclic compounds that have significant biological activity. There are examples of drug resistance modifiers [2], antioxidants [3], anticonvulsant [4], stress protective effect [5] and a drug for the treatment of urinary urge incontinence [6], among other properties of 1,4-DHPs. According to their pharmacological activity, the most well-known activity of 1,4-DHPs is their ability to act as calcium antagonists or calcium channel blockers [7-8]. The first step of the metabolism of 1,4-DHPs involves their oxidation into the corresponding pyridines. Pyridines are important as antitubercular, antibacterial [9], anti-inflammatory, antiasthmatic [10]

*Address correspondence to this author at theCentro de Investigación y Desarrollo en Ciencias Aplicadas "Dr. J.J. Ronco" (CINDECA), Departamento de Química, Facultad de Ciencias Exactas, UNLP-CCT-Conicet. Calles 47 N$^{\circ}$ 257, B1900AJK La Plata, Argentina; Tel: +54 0221421 1353; E-mail:1ms@quimica.unlp.edu.ar and antidepressant agents [11]. Accordingly, several procedures have been developed in order to study the oxidative dehydrogenation of 1,4-DHPs.

It has been proved that the introduction of vanadium into the Keggin framework is beneficial for redox catalysis, shifting its activity from acid to redox-dominated [12]. This can be explained through the energy gap between the highest occupied molecular orbital (HOMO) and the lowest unoccupied molecular orbital (LUMO), which is correlated with the reduction potential of the HPCs. It has been demonstrated that the more reducible HPCs have the smaller energy gap between the HOMO and the LUMO. It has also been reported that vanadium-containing HPCs show excellent redox properties because the substitution of vanadium stabilizes the LUMOs [13]. Continuing with our studies on HPCs synthesis and their use as effective, reusable and stable solid catalysts, we prepared and characterized vanadium (V) substituted Wells-Dawson heteropolysalt. Also, we performed the oxidation of a 1,4-DHP in acetonitrile media with $\mathrm{H}_{2} \mathrm{O}_{2}$ as oxidant agent using the HPC.

\section{EXPERIMENTAL}

\subsection{Synthesis of Catalysts}

\subsubsection{Tungstenphosphate Potassium salt $\left(\mathrm{K}_{6} \mathrm{P}_{2} W_{18} \mathrm{O}_{62} \cdot 10\right.$ $\mathrm{H}_{2} \mathrm{O}$ )}

The unsubstituted potassium salt catalyst (WDK) was synthesized according to the following procedure: 


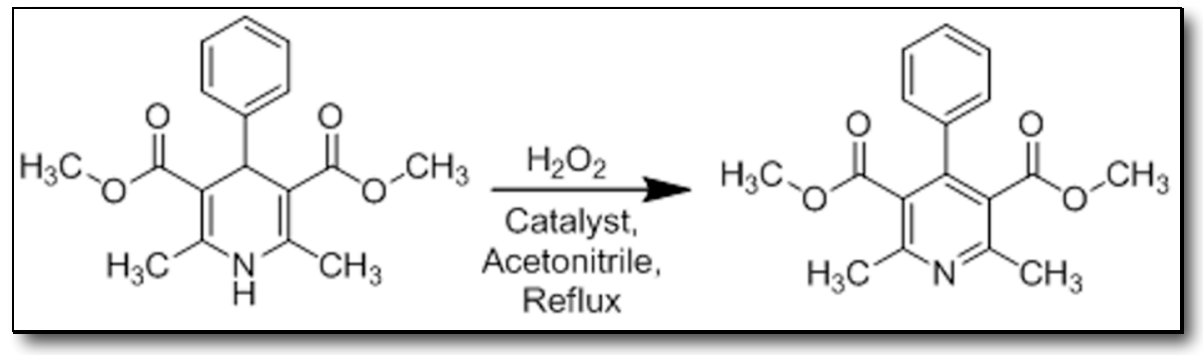

Scheme (1). General reaction taken as aim.

The $\alpha / \beta \quad \mathrm{K}_{6} \mathrm{P}_{2} \mathrm{~W}_{18} \mathrm{O}_{62} .10 \quad \mathrm{H}_{2} \mathrm{O}$ isomer mixture was prepared according to the method reported by Baronetti et al. [14]: to a boiling solution of $\mathrm{Na}_{2} \mathrm{WO}_{4} \cdot 2 \mathrm{H}_{2} \mathrm{O}$ concentrated $\mathrm{H}_{3} \mathrm{PO}_{4}$ was added in a 4:1 acid/salt molar ratio. This mixture was boiled continuously for $8 \mathrm{~h}$. Then, $\mathrm{KCl}$ was added in order to precipitate the salt. The solid thus obtained was purified by recrystallization and cooled overnight at $5^{\circ} \mathrm{C}$.

\subsubsection{Vanadotungstenphosphate Potassium Salt $\left(\alpha_{2}-1-\right.$ $\left.\mathrm{K}_{7} \mathrm{P}_{2} \mathrm{VW}_{17} \mathrm{O}_{61} \cdot 18 \mathrm{H}_{2} \mathrm{O}\right)$}

The V-substituted potassium salt catalyst (WDKV) was synthesized according to the following procedure.

1. The $\alpha / \beta \mathrm{K}_{6} \mathrm{P}_{2} \mathrm{~W}_{18} \mathrm{O}_{62} .10 \mathrm{H}_{2} \mathrm{O}$ isomer mixture was prepared according to the method reported by Baronetti et al. [14] as described in the previous section.

2. The white solid lacunar heteropolyoxoanion $\alpha_{2}$ $\mathrm{K}_{10} \mathrm{P}_{2} \mathrm{~W}_{17} \mathrm{O}_{61} .15 \mathrm{H}_{2} \mathrm{O}$ was synthesized from this $\alpha / \beta$ $\mathrm{K}_{6} \mathrm{P}_{2} \mathrm{~W}_{18} \mathrm{O}_{62} .10 \mathrm{H}_{2} \mathrm{O}$ isomer mixture according to the technique reported by Briand et al. [15]: the green $\alpha / \beta$ isomer mixture was treated with a $\mathrm{KHCO}_{3}$ solution at $40^{\circ} \mathrm{C}$ under stirring. The resultant white precipitate was purified by recrystallization and cooled overnight at $5^{\circ} \mathrm{C}$. The crystals so obtained were washed with pure ethanol.

3. The monosubstituted sample $1-\mathrm{K}_{7} \mathrm{P}_{2} \mathrm{VW}_{17} \mathrm{O}_{62} \cdot 18 \mathrm{H}_{2} \mathrm{O}$ was synthesized from $\alpha_{2}-\mathrm{K}_{10} \mathrm{P}_{2} \mathrm{~W}_{17} \mathrm{O}_{61} .15 \mathrm{H}_{2} \mathrm{O}$ according to Briand et al. [15]: a hydrochloric solution of the lacunar heteropolyoxoanion was treated with $\mathrm{NaVO}_{3}$. Subsequently $\mathrm{KCl}$ was added to this solution and a precipitate appeared. The orange crystals were washed with distilled water.

\subsection{Catalyst Characterization}

Fresh solid samples were characterized by ${ }^{31} \mathrm{P}$ MAS-NMR measurements in Bruker Avance II-300 equipment, using $85 \% \mathrm{H}_{3} \mathrm{PO}_{4}$ as an external standard under the following operation conditions: pulse width of $\pi / 2{ }^{31} \mathrm{P}: 5 \mu \mathrm{s}$; repetition time $\left({ }^{31} \mathrm{P}\right)$ : $60 \mathrm{~s}$; rotation speed: $7 \mathrm{KHz}$. FTIR measurements were made in Bruker IFS 66 equipment. Besides, the morphology of the compounds was analyzed by scanning electron microscopy (SEM) with Philips SEM 505 microscope. X-ray diffraction (XRD) spectra for $2 \theta$ values between $5^{\circ}$ and $45^{\circ}$ were recorded in Philips PW 1732 equipment. The following operating conditions were used: source voltage, 40 $\mathrm{kV}$; source current, $20 \mathrm{~mA}$; goniometer speed, $\Delta \theta=2 \% \mathrm{~min}$; chart speed, $2 \mathrm{~cm} / \mathrm{min}$; slit, $1 / 0.1 / 1^{\circ}$. Thermogravimetric analyses (TGA) were carried out in Shimadzu TGA-50H equipment. Samples were heated in air from room tempera- ture to $800^{\circ} \mathrm{C}$ at a rate of $10^{\circ} \mathrm{C} / \mathrm{min}$. Potentiometric titration measurements were made in Metrohm 794 Basic Titrino equipment by titrating acetonitrile catalyst solutions with $n$ butylamine.

\subsection{Catalytic Tests}

The general procedure for the oxidation of 1,4-DHPs was developed from the results of the following experiments. The effect of catalyst, amount of oxidant agent, reaction temperature and amount of catalyst on reaction conversion and its selectivity towards desired product was analyzed. With that purpose, the general reaction presented in Scheme (1) was performed. The starting 1,4-DHP was prepared according to Sathicq et al. [16]. Reactions were monitored by gas chromatography (GC), with Shimadzu GC-2014 equipment. The conversion was defined as the ratio of converted species to the initial concentration. The products were identified by comparison of physical data (TLC, NMR) with those reported [17]. ${ }^{13} \mathrm{C}-\mathrm{NMR}$ and ${ }^{1} \mathrm{H}-\mathrm{NMR}$ spectra were recorded at room temperature on Bruker AC 200 using tetramethylsilane (TMS) as internal standard. Decomposition of $\mathrm{H}_{2} \mathrm{O}_{2}$ was evaluated through iodimetric method.

\section{RESULTS AND DISCUSSION}

\subsection{Catalyst Characterization}

Fig. (1) shows the infrared spectroscopic results for the lacunar heteropolyoxoanion, WDK and WDKV catalysts. In the case of the lacunar heteropolyoxoanion, the typical signals expected for this structure were found: $740,805,880$, $905,940,985,1022$ and $1084 \mathrm{~cm}^{-1}[14,18]$. For WDK and WDKV samples, Fig. (1) shows the typical signals expected for the Wells-Dawson structure: $\mathrm{PO}_{4}$ tetrahedron (1089 $\left.\mathrm{cm}^{-1}\right), \mathrm{W}=\mathrm{O}\left(950 \mathrm{~cm}^{-1}\right)$ and $\mathrm{W}-\mathrm{O}-\mathrm{W}\left(912\right.$ and $\left.779 \mathrm{~cm}^{-1}\right)$ bonds $[14,15]$. Therefore it is possible to assume that the Vsubstituted catalyst keeps the corresponding Wells-Dawson structure.

In addition, the Wells-Dawson structure known as $\alpha$ isomer possesses two identical "half units" $\mathrm{XM}_{9} \mathrm{O}_{31}$ of the central atom surrounded by nine octahedral units linked through oxygen atoms. The $\alpha$-isomer of Wells-Dawson potassium salt has two equivalent phosphorus atoms and consequently, it shows only one peak in the ${ }^{31} \mathrm{P}-\mathrm{NMR}$ spectrum at -12.3 ppm. Fig. (2) shows the ${ }^{31} \mathrm{P}$ MAS-NMR spectrum of the monosubstituted $\mathrm{V}$ compound (WDKV) synthesized in this work. It can be observed that WDKV sample shows a peak with a chemical shift of $-13.4 \mathrm{ppm}$, corresponding to the less perturbed $\mathrm{P}$ atom, and another one with a chemical shift of $-11.3 \mathrm{ppm}$, in agreement with previous literature re- 


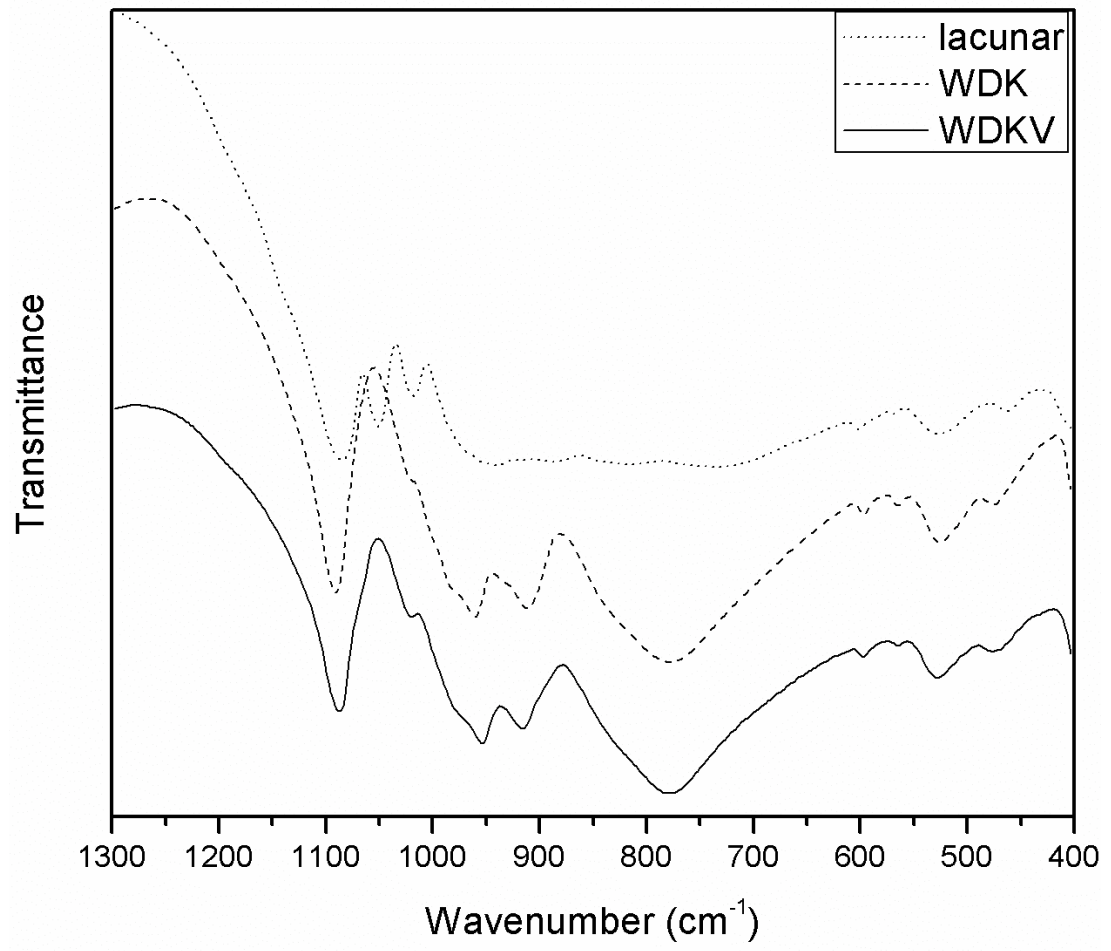

Fig. (1). FTIR spectra of fresh lacunar heteropolyoxoanion, WDK and WDKV.

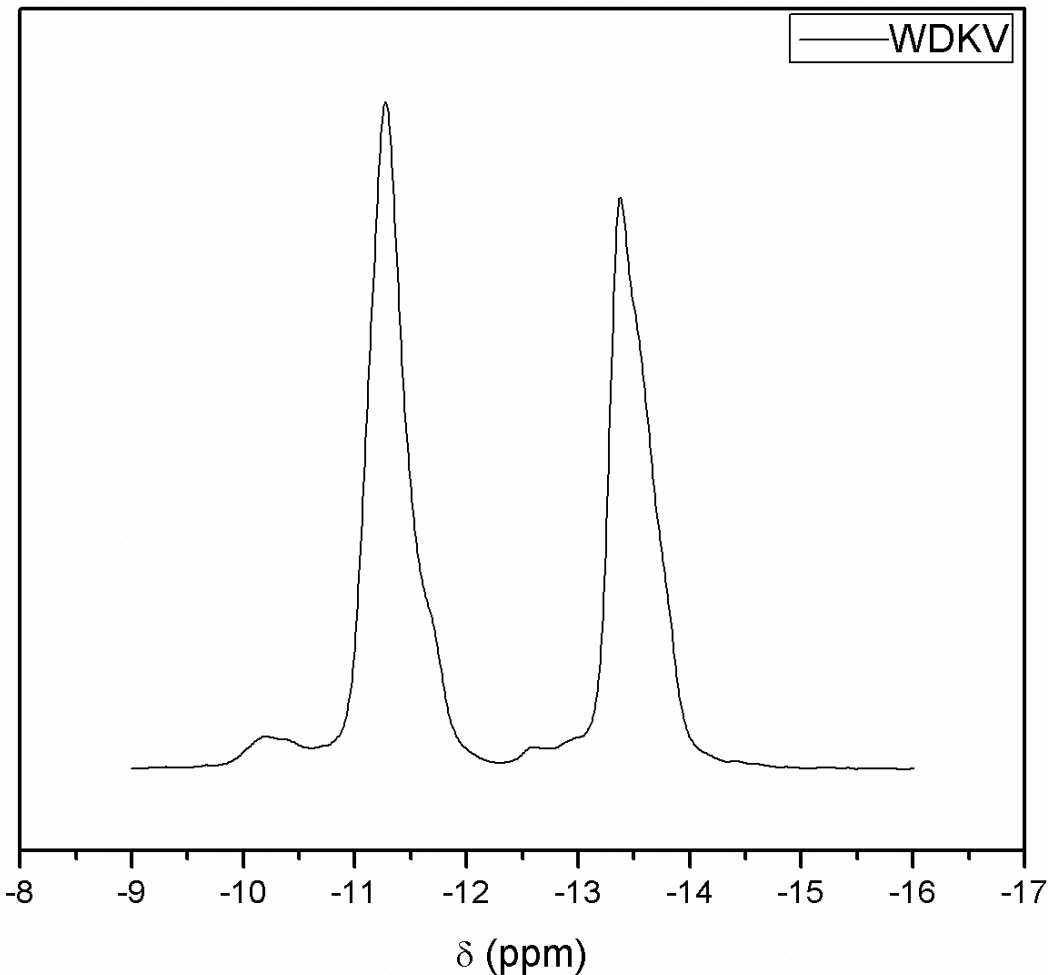

Fig. (2). ${ }^{31} \mathrm{P}-\mathrm{NMR}$ spectra of fresh WDKV.

ports for vanadium incorporation into a polar position of the $\mathrm{PW}_{8}$ half [18]. These results indicate that the $\mathrm{V}$ atom has been successfully included in the Wells-Dawson structure.

Fig. (3) depicts a SEM micrograph of fresh vanadium substituted and unsubstituted Wells-Dawson potassium salts.
Both samples show a smooth morphology, in contrast to the rough texture obtained for other heteropolyacids with Keggin structure [15].

Moreover, Fig. (4) shows the X-ray patterns of fresh WDK and WDKV. Fig. (4) shows intense signals at 6.6, 7.2, 


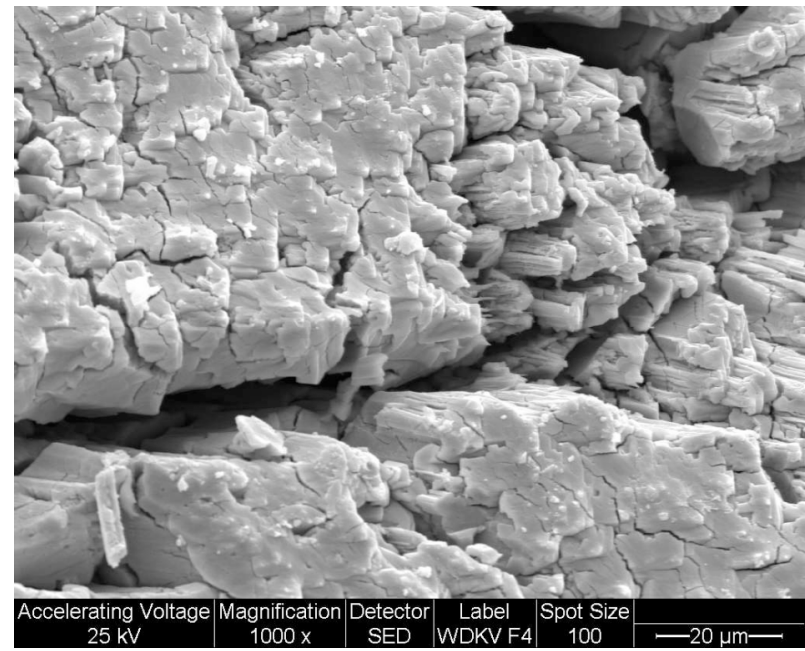

a

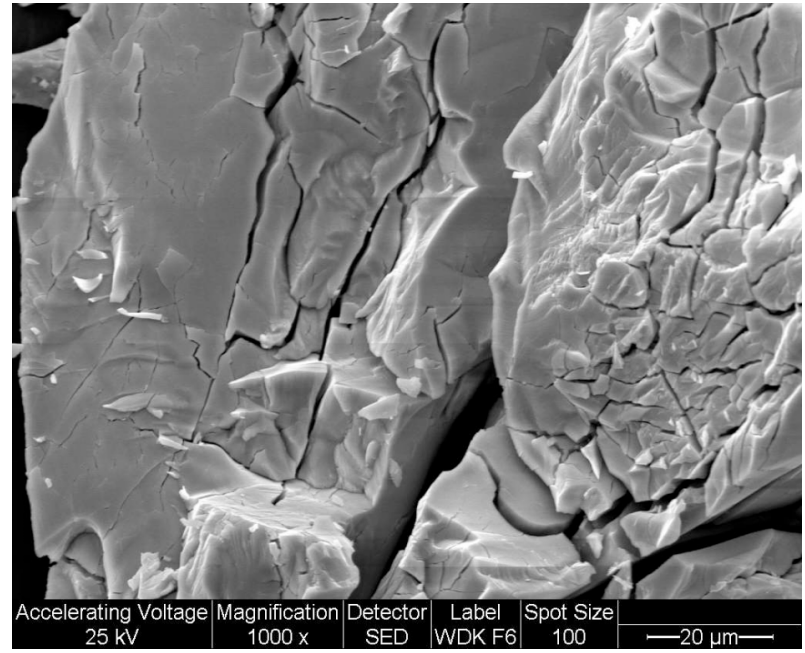

b

Fig. (3). Scanning electron photomicrographs of fresh: a) WDKV and b) WDK (Magnification x 1000).

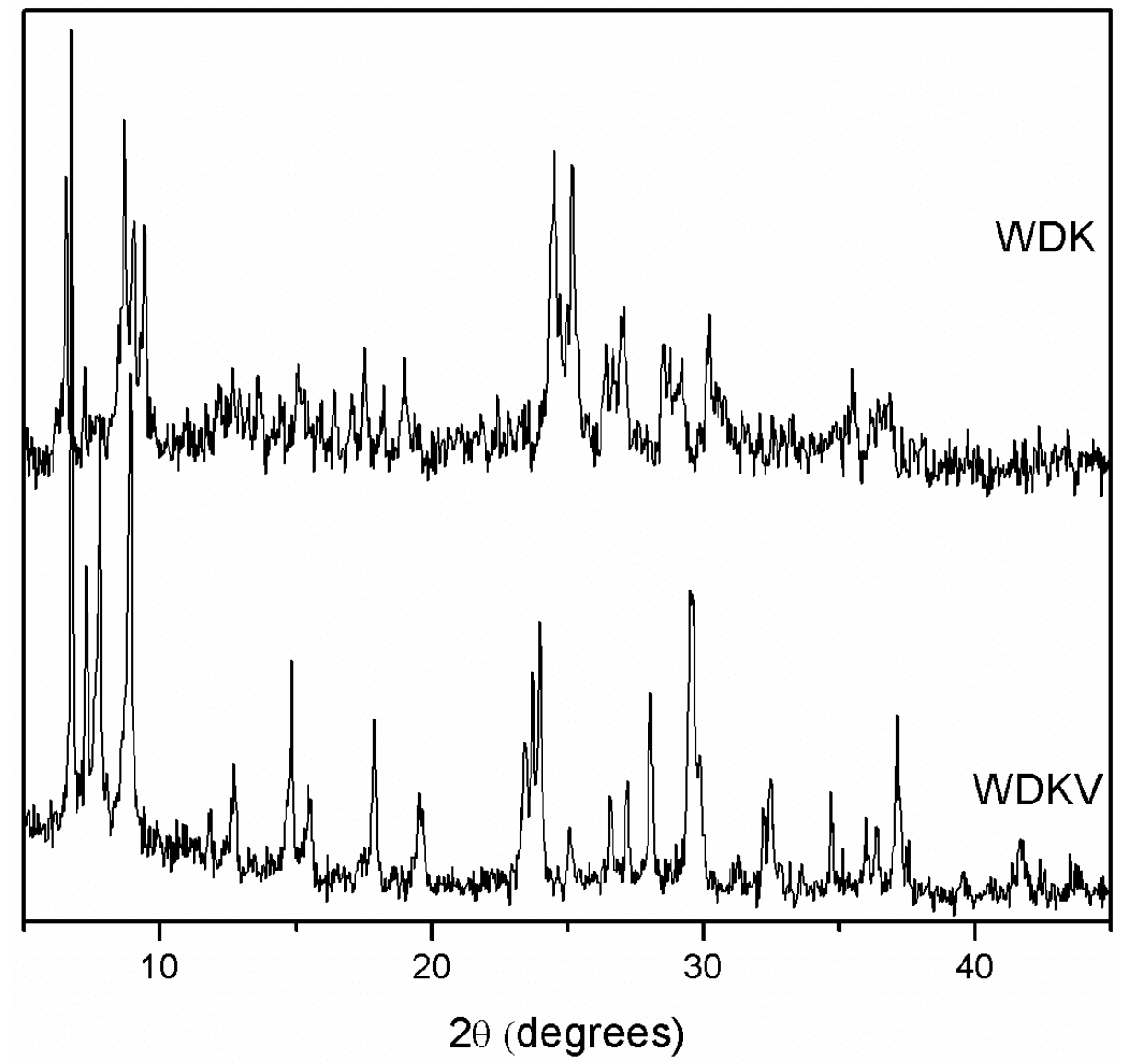

Fig. (4). DRX diffractogram of fresh WDK and WDKV.

$8.7,9.0,9.4,15.1,17.5,18.2,19.0,24.5,25.2,26.4,26.7$, $27.1,28.6,28.8,29.2,30.2$ and 35.5 which coincide with those reported for the $\alpha / \beta$-isomer mixture of the $\mathrm{K}_{6} \mathrm{P}_{2} \mathrm{~W}_{18} \mathrm{O}_{62} .10 \mathrm{H}_{2} \mathrm{O}$ Dawson heteropolyoxoanion [15]. Fig. (4) also shows some main diffraction peaks at: 6.7, 7.3, 7.8, $8.9,12.7,14.9,15.4,17.8,19.5,23.4,23.7,24.0,25.1,26.5$, $27.2,28.1,29.5,32.5,34.7,36.0,36.4,37.1$ and 37.2 for WDKV sample. The mentioned diffraction peaks coincide with those reported by Briand et al. for $1-\mathrm{K}_{7} \mathrm{P}_{2} \mathrm{VW}_{17} \mathrm{O}_{62} .18$ -
$\mathrm{H}_{2} \mathrm{O}$ [15]. These data also indicated that WDKV keeps the Wells-Dawson structure after vanadium substitution.

By TGA we determined the number of water molecules involved in WDK and WDKV structures. A weight loss step was detected between room temperature and $280^{\circ} \mathrm{C}$, for both compounds. From $280^{\circ} \mathrm{C}$ to $800^{\circ} \mathrm{C}$ no additional weight loss was observed. These results indicated that WDKV and WDK possess 10 and $7 \mathrm{H}_{2} \mathrm{O}$ hydration molecules, respectively. 


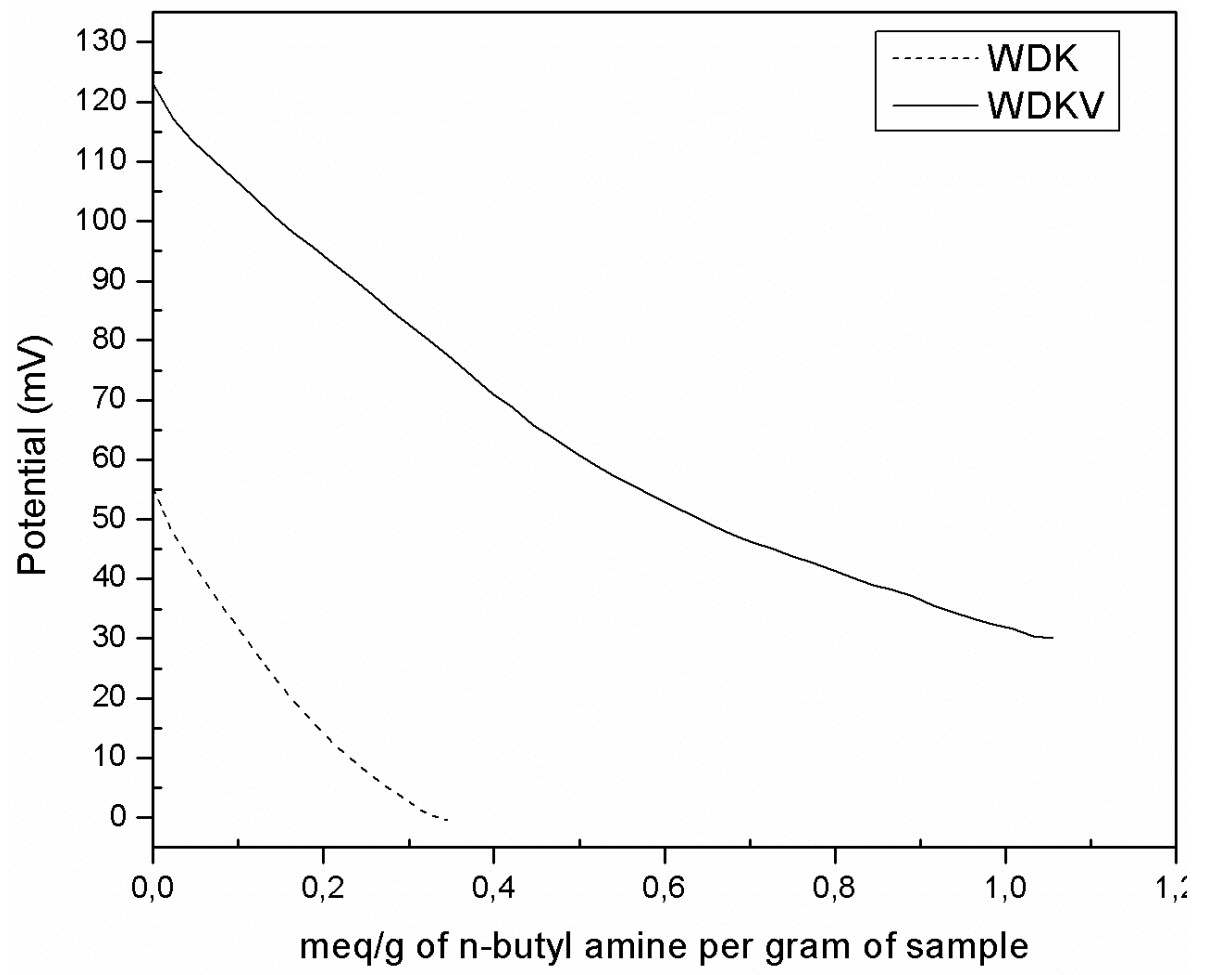

Fig. (5). Potentiometric titration of n-butyl amine with WDK and WDKV.

The acidic properties of the solid acid catalysts were evaluated by potentiometric titration with n-butylamine. The maximum acid strength of superficial sites is indicated by the initial electrode potential (E). According to this, the acid strength of the superficial sites could be classified by using the following range: very strong site: $\mathrm{E}>100 \mathrm{mV}$; strong site: $0<\mathrm{E}<100 \mathrm{mV}$; weak site: $-100<\mathrm{E}<0 \mathrm{mV}$; and very weak site: $\mathrm{E}<-100 \mathrm{mV}$ [19]. By plotting the potential derivative obtained versus n-butyl amine solution volume, it is possible to determine the number of different acidic sites observing the number of peaks that appear on the graph. Moreover, the area under each curve gives us information about the number of acidic sites in the catalyst.

According to the results obtained through potentiometric titration by titrating n-butyl amine, WDKV has very strong acidic sites $(\mathrm{E}>100 \mathrm{mV})$ and WDK has strong acidic sites $(0$ $<\mathrm{E}<100 \mathrm{mV}$ ). As a result, WDKV has more acidic sites than WDK, and they are a little more acid than WDK sites (Fig. 5).

\subsection{Catalytic Tests}

Physical data of the oxidized product were analyzed (TLC, NMR). By comparison between reported and experimental ${ }^{13} \mathrm{C}$-NMR and ${ }^{1} \mathrm{H}$-NMR data, we conclude that the desired product was obtained. No secondary products were observed. In addition, decomposition of $\mathrm{H}_{2} \mathrm{O}_{2}$ is minimal in the reactions conditions.

In order to study the effect of the catalyst, the comparison between the use of WDK, WDKV and no catalyst was made (Fig. 6). As we can see the conversion is higher using WDKV catalyst.
Moreover, the preferable amount of catalyst (Fig. 7) was studied. We found that there is no significant difference between 1 and $3 \mathrm{mmol} \%$. Therefore, $1 \mathrm{mmol} \%$ is the optimal amount for our system.

The optimal ratio between 1,4-DHP and $\mathrm{H}_{2} \mathrm{O}_{2}$ was selected by carrying out some experiences with different quantities of the oxidant agent (Fig. 8). There is no significant difference by using quantities of oxidant agent larger than $1: 214$. So, we consider that a ratio $1: 214$ is appropriate to achieve excellent conversion values.

The last parameter that we tested is the effect of reaction temperature on reactant conversion, and it was tested in a range from 323 to $354 \mathrm{~K}$ (Fig. 9). As is shown, in this system the best option for work temperature is at reflux of the selected reaction solvent. In terms of the difference between $354 \mathrm{~K}$ curve shape and the other ones, it is possible to relate it to high reaction activation energy. The higher is activation energy, the stronger is the temperature dependence of the rate constant [20].

It could be noted that Figs. (7 to 9) present an induction period at short reaction times. It is possible to explain this effect through the species that participate in the oxidation reaction. Reactive species between the heteropolyoxotungstate and hydrogen peroxide must be formed until the desired reaction proceeds [21].

The optimal procedure is the following: $1 \mathrm{mmol} \%$ of WDKV, a ratio 1,4-DHP: $\mathrm{H}_{2} \mathrm{O}_{2}(1: 214)$ at reflux of acetonitrile. 


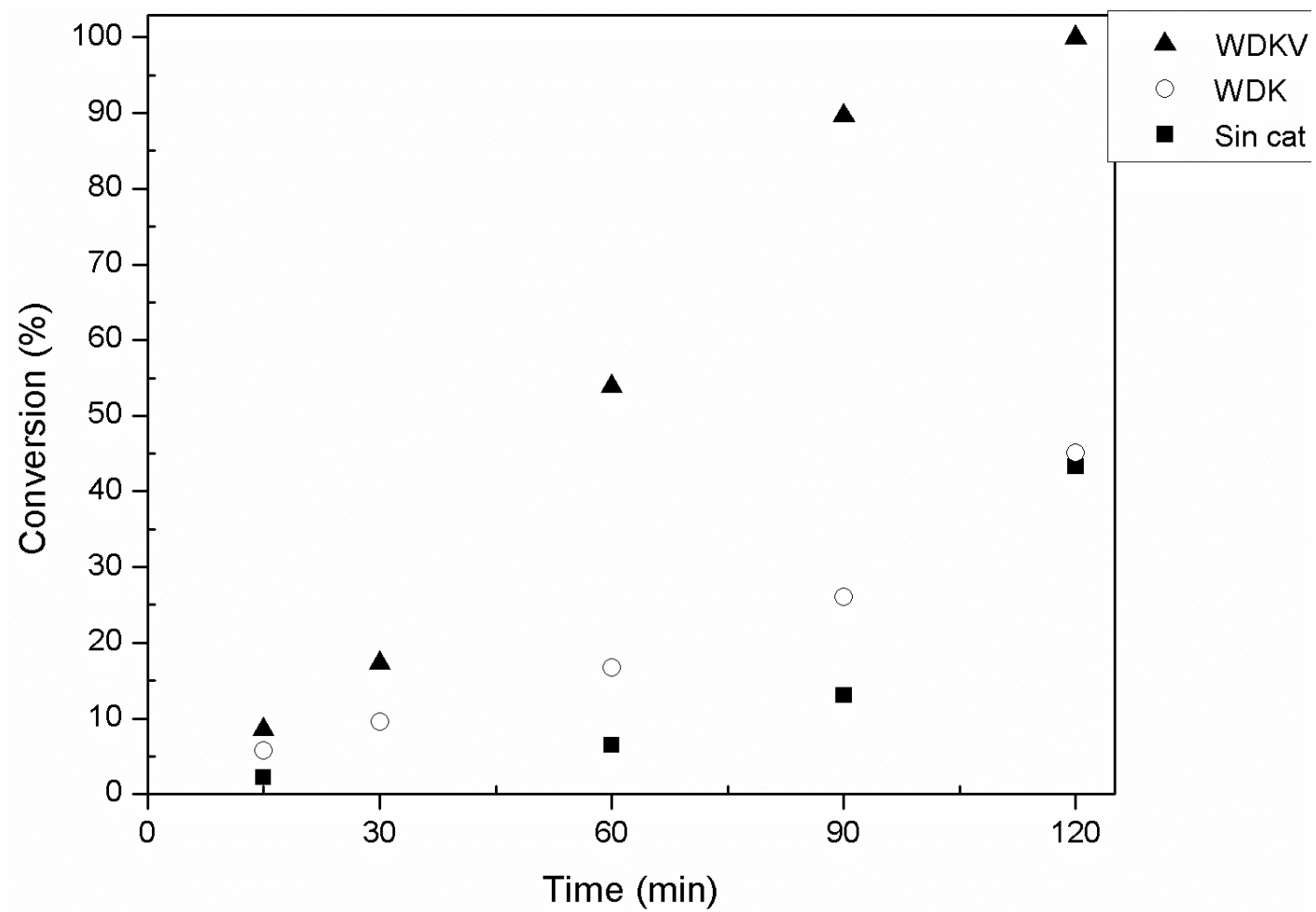

Fig. (6). Selection of the optimal catalyst.

Reaction conditions: 3 mmol \% of catalyst, 1,4-DHP: $\mathrm{H}_{2} \mathrm{O}_{2}(1: 214)$, reflux in acetonitrile.

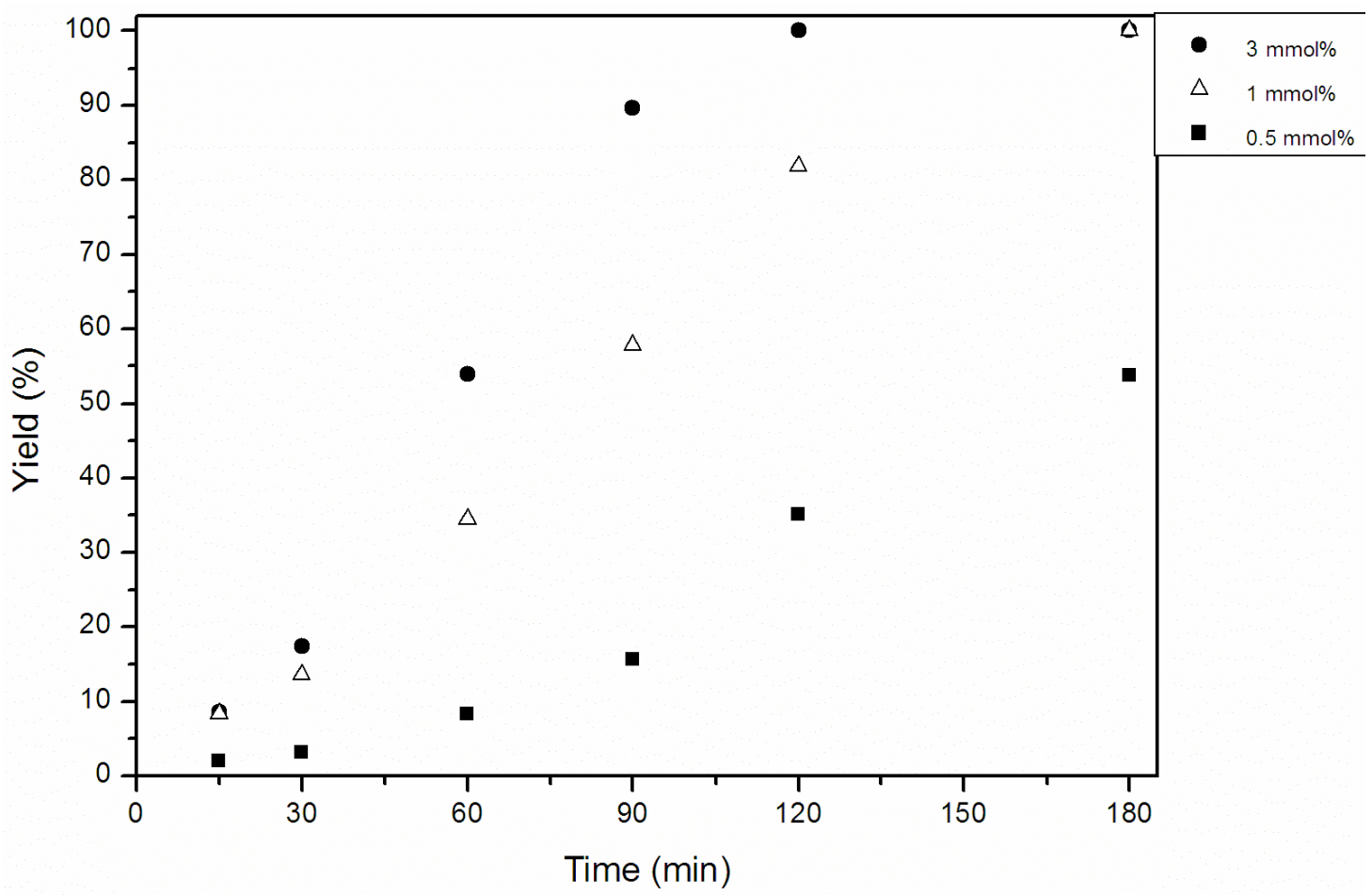

Fig. (7). Selection of the optimal amount of catalyst.

Reaction conditions: WDKV (varying from 0.5 to 3 mmol\%), 1,4-DHP: $\mathrm{H}_{2} \mathrm{O}_{2}$ (1:214), reflux in acetonitrile. 


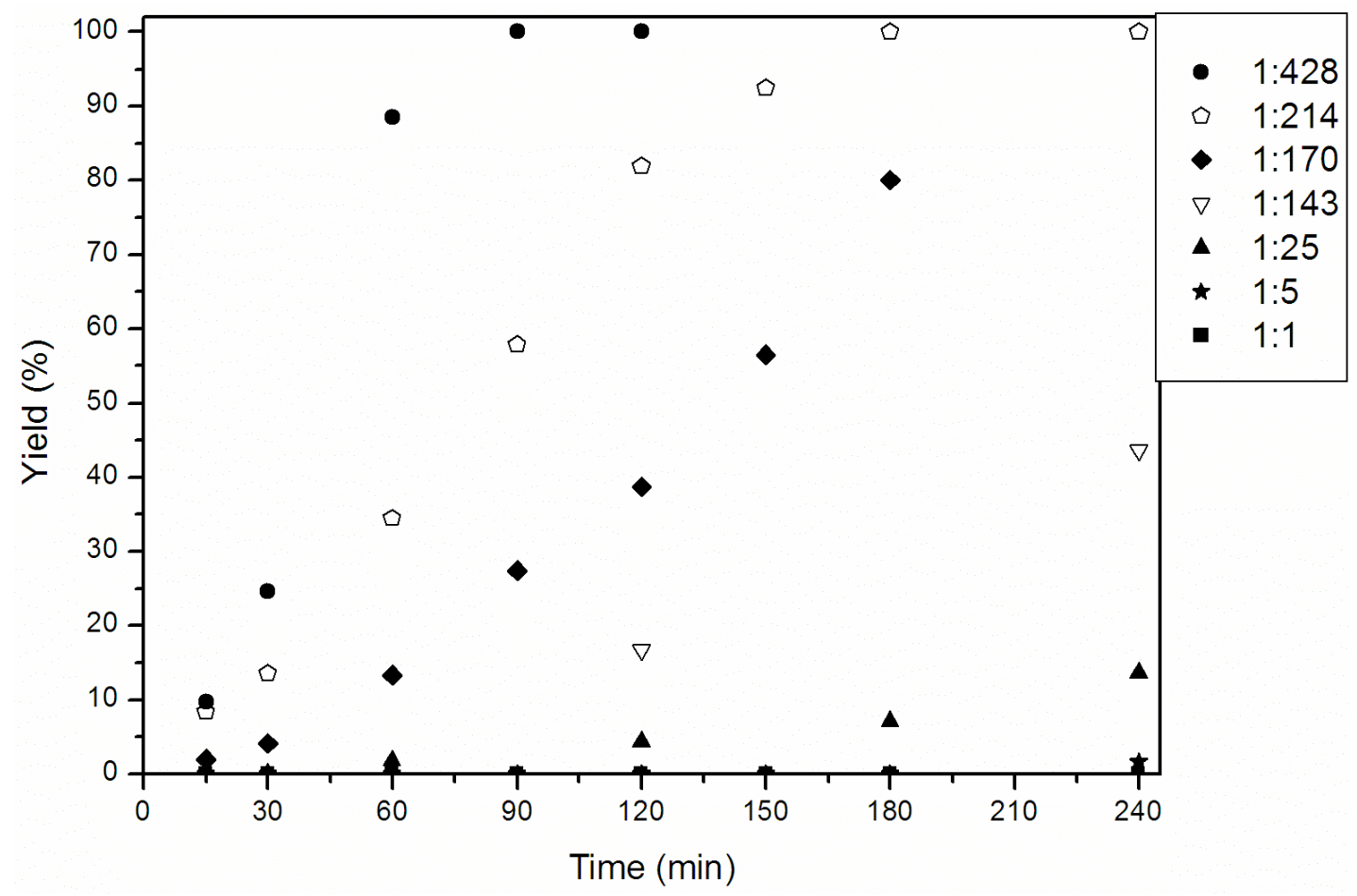

Fig. (8). Selection of the optimal ratio 1,4-DHP: $\mathrm{H}_{2} \mathrm{O}_{2}$.

Reaction conditions: $1 \mathrm{mmol} \%$ of WDKV, 1,4-DHP: $\mathrm{H}_{2} \mathrm{O}_{2}$ (varying between 1:1 and 1:428), reflux in acetonitrile.

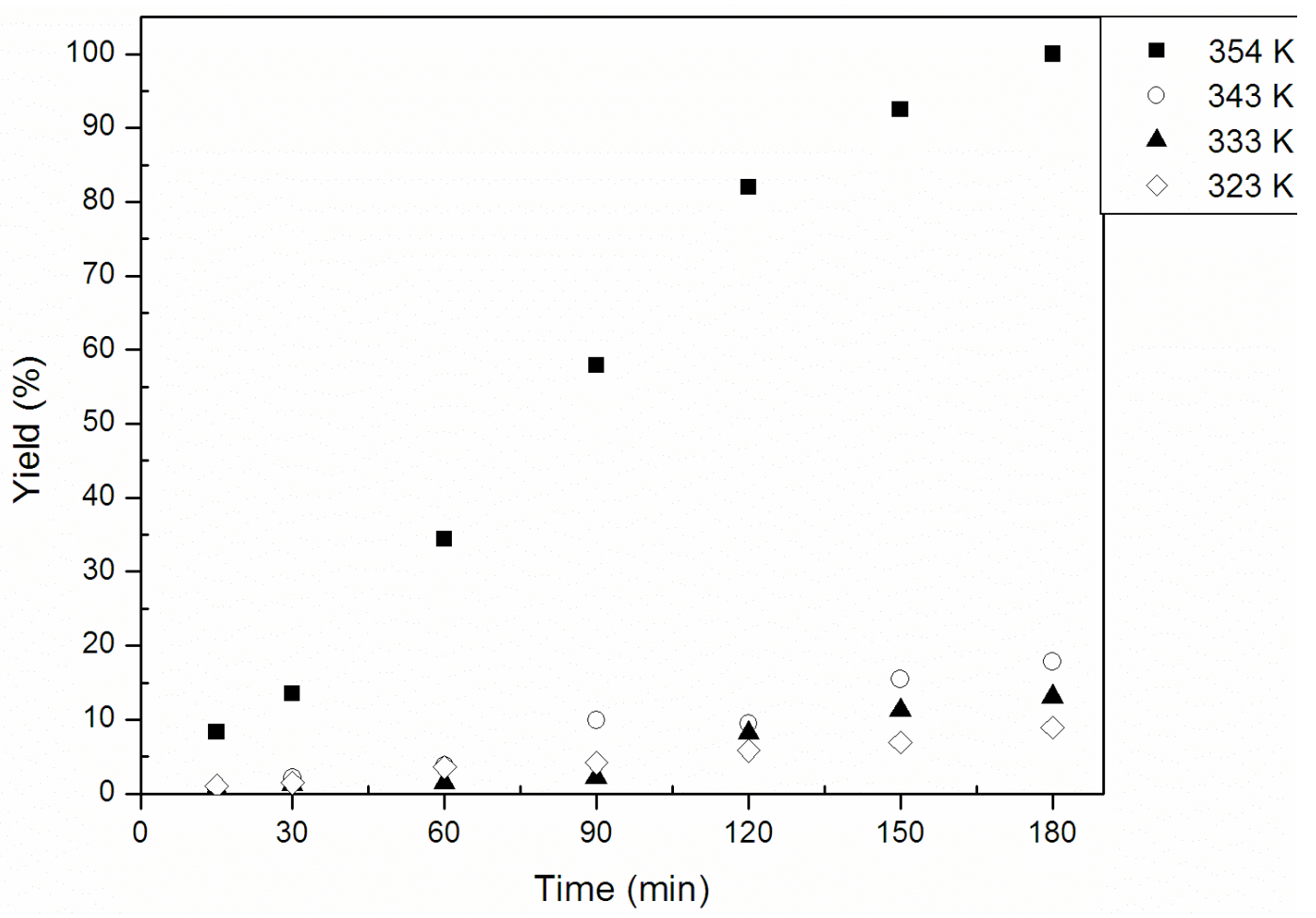

Fig. (9). Study of the effect of reaction temperature.

Reaction conditions: $1 \mathrm{mmol} \%$ of WDKV, 1,4-DHP: $\mathrm{H}_{2} \mathrm{O}_{2}$ (1:214), acetonitrile and temperature varying from 323 to $354 \mathrm{~K}$.

\section{CONCLUSIONS}

An effective route for the reaction on oxidation of 1,4DHPs was developed and analyzed. We prepared a WellsDawson potassium salt with a V substitution (WDKV). Also, we characterized this catalyst and compared the results with those from the literature and with another known WellsDawson potassium salt (WDK). It is possible to conclude that the introduction of $\mathrm{V}$ was performed successfully, and the product maintains Wells-Dawson structure. Then, the 
performances of both catalysts were compared in a desired oxidation reaction of a 1,4-DHP. As WDKV shows better conversion results, we selected this catalyst and looked for an optimal procedure to oxidize a 1,4-DHP by using an important excess of $\mathrm{H}_{2} \mathrm{O}_{2}$ in acetonitrile. It must be noted that along all reactions, no secondary products were observed.

\section{CONFLICT OF INTEREST}

The authors confirm that this article content has no conflicts of interest.

\section{ACKNOWLEDGEMENTS}

The authors thank CONICET, Agencia Nacional de Promoción Científica y Tecnológica (Argentina), Comisión de Investigaciones Científicas (CIC), and Universidad Nacional de La Plata for financial support. AGS, GTB and HJT are members of CONICET.

\section{REFERENCES}

[1] Cavani, F. Heteropolycompound-based catalysts: a blend of acid and oxidizing properties. Cat. Today, 1998, 41, 73-86.

[2] Sridhar, R.; Perumal, P. T. A new protocol to synthesize 1,4dihydropyridines by using 3,4,5-trifluorobenzeneboronic acid as a catalyst in ionic liquid: synthesis of novel 4-(3-carboxyl- $1 \mathrm{H}$ pyrazol-4-yl)-1,4-dihydropyridines. T. Tetrahedron, 2005, 61, 2465-2470.

[3] Heravi, M. M.; Behbahani, F. K.; Oskooie, H. A.; Shoar, R. H. Catalytic aromatization of Hantzsch 1,4-dihydropyridines by ferric perchlorate in acetic acid. Tetrahedron Lett., 2005, 46, 2775- 2777.

[4] Tussel, J. M.; Barron S.; Seratosa J. Anticonvulsant activity of $\mathrm{HCH}$, calcium channel blockers and calmodulin antagonists in seizures induced by lindane and other convulsant drug. Brain Res., 1993, 622, 99-104.

[5] Tussel, J. M.; Barron, S.; Seratosa, J. Anticonvulsant activity of $\delta$ $\mathrm{HCH}$, calcium channel blockers and calmodulin antagonists in seizures induced by lindane and other convulsant drugs. Brain Res., 1993, 622, 99-104.

[6] Tarasenko, L. M.; Neporada, K. S.; Klusha, V. Stress-Protective Effect of Glutapyrone Belongingto a New Type of Amino AcidContaining 1,4-Dihyropyridines on Periodontal Tissuesand Stom- ach in Rats with Different Resistance to Stress. Bull. Exp. Biol. and Med., 2002, 133, 426-428.

[7] Moseley, J. D. Alternative esters in the synthesis of ZD0947. Tetrahedron Lett., 2005, 46, 3179-3181.

[8] Eisner, U.; Kuthan, J. The Chemistry of Dihydropyridines. Chem. Rev., 1972, 72, 1-42.

[9] Henry, G. H. Tetrahedron, 2004, 60, 6043-6061.

[10] Li, A. H.; Moro, S.; Forsyth, N.; Melman, N.; Ji, X.; Jacobson, K. A. J. Med. Chem., 1999, 42, 706-721.

[11] Vacher, B.; Bonnaud, B.; Funes, P.; Jubault, N.; Koek, W.; Assié, M. B.; Cosi, C.; Kleven. M. J. Med. Chem., 1999, 42, 1648-1660.

[12] Mizuno, N; Misono, M. Heteropolyanions in catalysis. J. Mol. Catal., 1994, 86, 319-342.

[13] Weber, R. S. Molecular orbital study of C-H bond breaking during the oxidative dehydrogenation of methanol catalyzed by metal oxide surfaces. J. Phys. Chem., 1994, 98, 2999-3005.

[14] Baronetti, G.; Briand, L.; Sedran, U.; Thomas, H. Heteropolyacidbased catalysis. Dawson acid for MTBE synthesis in gas phase. Appl. Catal. A: Gen., 1998, 172, 265-272.

[15] Briand, L. E.; Thomas, H. J.; Baronetti, G. T. Thermal stability and catalytic activity of Wells-Dawson tungsten heteropoly salts. Appl. Catal. A: Gen., 2000, 201, 191-202.

[16] Sathicq, A.; Romanelli, G.; Ponzinibbio, A.; Baronetti, G.; Thomas, H. An Efficient One-Step Hantzsch Multicomponent Synthesis of 1,4-Dihydropyridines Via a Wells-Dawson Heteropolyacid Catalyst Under Solvent-Free Conditions. Lett. Org. Chem., 2010, 7 , 511-518.

[17] Miyamura, H.; Maehata, K.; Kobayashi, S. In Situ Coupled Oxidation Cycle Catalyzed by Highly Active and Reusable Pt-Catalysts: Dehydrogenative Oxidation Reactions in the Presence of a Catalytic Amount of $o$-Chloranil Using Molecular Oxygen as the Terminal Oxidant. Chem. Commun., 2010, 46, 8052-8054.

[18] Harmalker, S.; Leparulo, M.; Pope, M. Mixed-valence chemistry of adjacent vanadium centers in heteropolytungstate anions. I. Synthesis and electronic structures of mono-, di-, and trisubstituted derivatives of .alpha.-octadecatungstodiphosphate $\left({ }^{6-}\right)$ ion (.alpha.$\left.\left[\mathrm{P}_{2} \mathrm{~W}_{18} \mathrm{O}_{62}\right]^{6-}\right)$. J. Am. Chem. Soc., 1983, 105, 4286-4292.

[19] Cid, R.; Pecchi, G. Potentiometric method for determining the number and relative strength of acid sites in colored catalysts. Appl. Cat., 1985, 14, 15-21.

[20] Ingeniería de las Reacciones Químicas; Levenspiel, O., Ed.; Repla, S.A.: México D. F., 1987; p 31.

[21] Nomiya, K.; Nemoto, Y.; Hasegawa, T.; Matsuoka, S. Multicenter active sites of vanadium-substituted polyoxometalate catalysts on benzene hydroxylation with hydrogen peroxide and two reaction types with and without an induction period. J. Mol. Catal. A: Chem., 2000, 152, 55-68. 